\title{
ERRATUM: “ULTRAVIOLET THROUGH FAR-INFRARED SPATIALLY RESOLVED ANALYSIS OF THE RECENT STAR FORMATION IN M81 (NGC 3031)” (ApJ, 648, 987 [2006])
}

Pablo G. Pérez-González, Robert C. Kennicutt, Jr., Karl D. Gordon, Karl A. Misselt, Armando Gil de Paz, Charles W. Engelbracht, George H. Rieke, George J. Bendo, Luciana Bianchi, Samuel Boissier,

Daniela Calzetti, Daniel A. Dale, Bruce T. Draine, Thomas H. Jarrett,

David Hollenbach, and Moire K. M. Prescott

Because some proof corrections were not carried properly to the final version, there are some errors in the text, figures, and Table 2 of our published article.

1. In the machine-readable data table for Table 2, the values for the $A(V)$ are incorrect. A correct version of the complete data table is available in the electronic edition of this erratum.

2. Figures 2, 3, and 5 are missing some data points; correct versions of these figures are given here.

3. In Equation (1) the $(\lambda)$ on the left-hand side should not be subscript; the correct form of the equation is:

$$
F_{\text {dust }}(\lambda)=\sum C^{i} \kappa^{i}(\lambda) B_{\lambda}\left(T_{\text {dust }}^{i}\right) .
$$

4. In the text following equation (1), in the sentence "We assumed temperature ranges of 200-750, 35-100, and 10-25 for the aromatics, warm, and cold silicates, respectively," these ranges should have units of K.

5. Equation (13) has an extraneous + sign on the second line; this equation should read:

$$
\begin{aligned}
\log [L(8-1000)]= & (-0.14 \pm 0.30) \\
& +(1.050 \pm 0.041) \log [L(160)] .
\end{aligned}
$$

The Press sincerely apologizes for these errors.

Online material: machine-readable table

TABLE 2

Positions and Photometry for the Regions Selected at $24 \mu \mathrm{m}$ Resolution

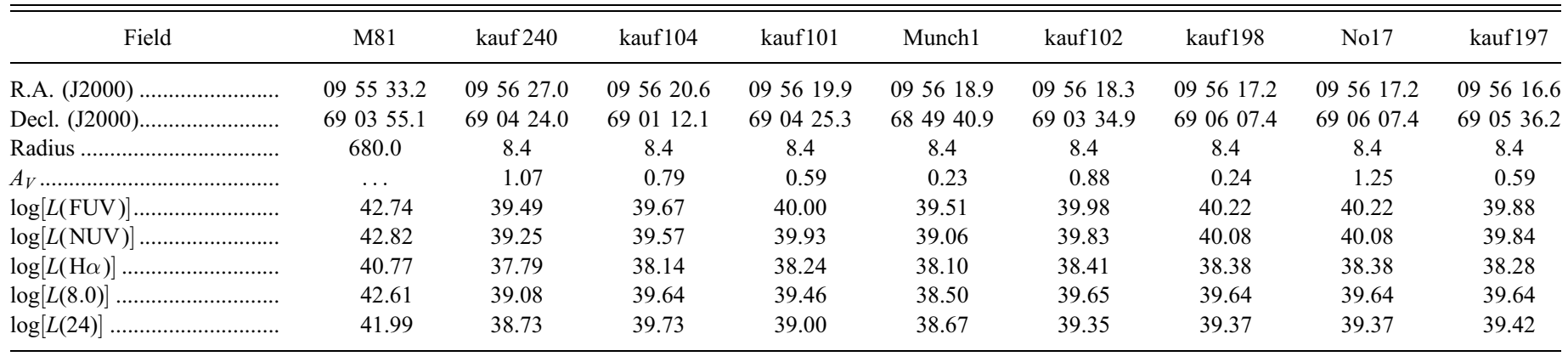

Note.-Table 2 is published in its entirety in the electronic edition of the Astrophysical Journal. A portion is shown here for guidance regarding its form and content. Units of right ascension are hours, minutes, and seconds, and units of declination are degrees, arcminutes, and arcseconds. The radius of each region is given in arcsec. All luminosities refer to observed values corrected for Galactic extinction (but no internal extinction corrections have been applied) and the units are ergs $\mathrm{s}^{-1}$. 


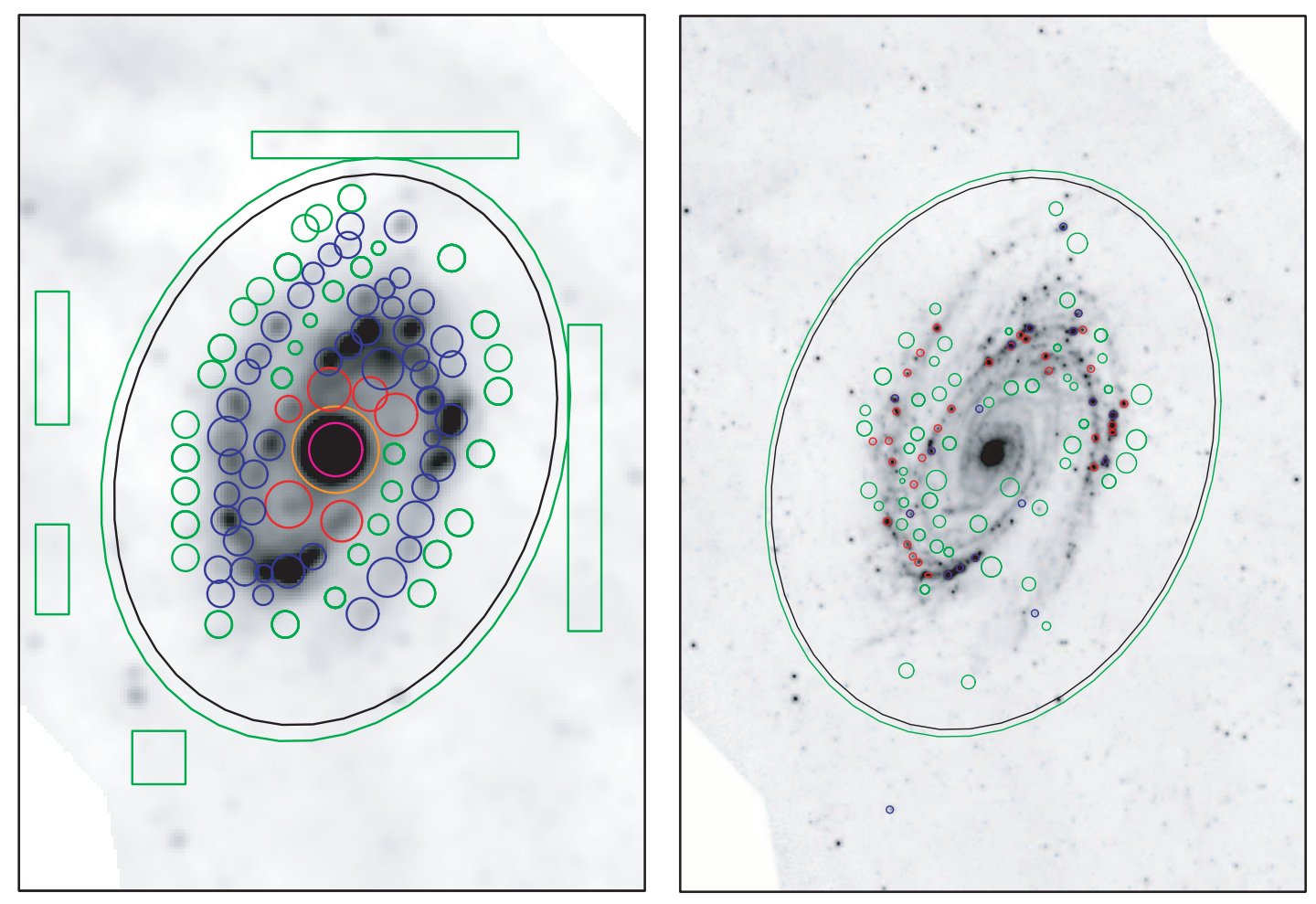

FIG. 2.- Spectral energy distributions for the entire M81 galaxy (top left panel), the nucleus (top right panel), and two regions in the arms (bottom panels) selected at 160RES, corresponding to the photometry estimated with the global sky subtraction. The open circles in the SED of the entire galaxy refer to several photometric points extracted from NED (for apertures nearly as large as ours): three UV/optical fluxes from Code \& Welch (1982) and de Vaucouleurs et al. (see footnote 12), the three NIR fluxes from 2MASS (Jarrett et al. 2000), the flux in the $N$-band from Kleinmann \& Low (1970), and the four IRAS fluxes measured by Rice et al. (1988) and Devereux et al. (1995). Horizontal error bars for each photometry point show the width of the used filter. The data have been fitted (red line) to a model of the emission of the stars (a single burst with a exponential SFR), the gas (including the most common emission lines, shown in the plot with a width equal to the resolution of the stellar model at each wavelength), and the dust (including two dust components of two different temperatures, and aromatic molecules). Our models of the dust emission are compared with the ones carried out following the method in Draine et al. (2006, in preparation; green line).
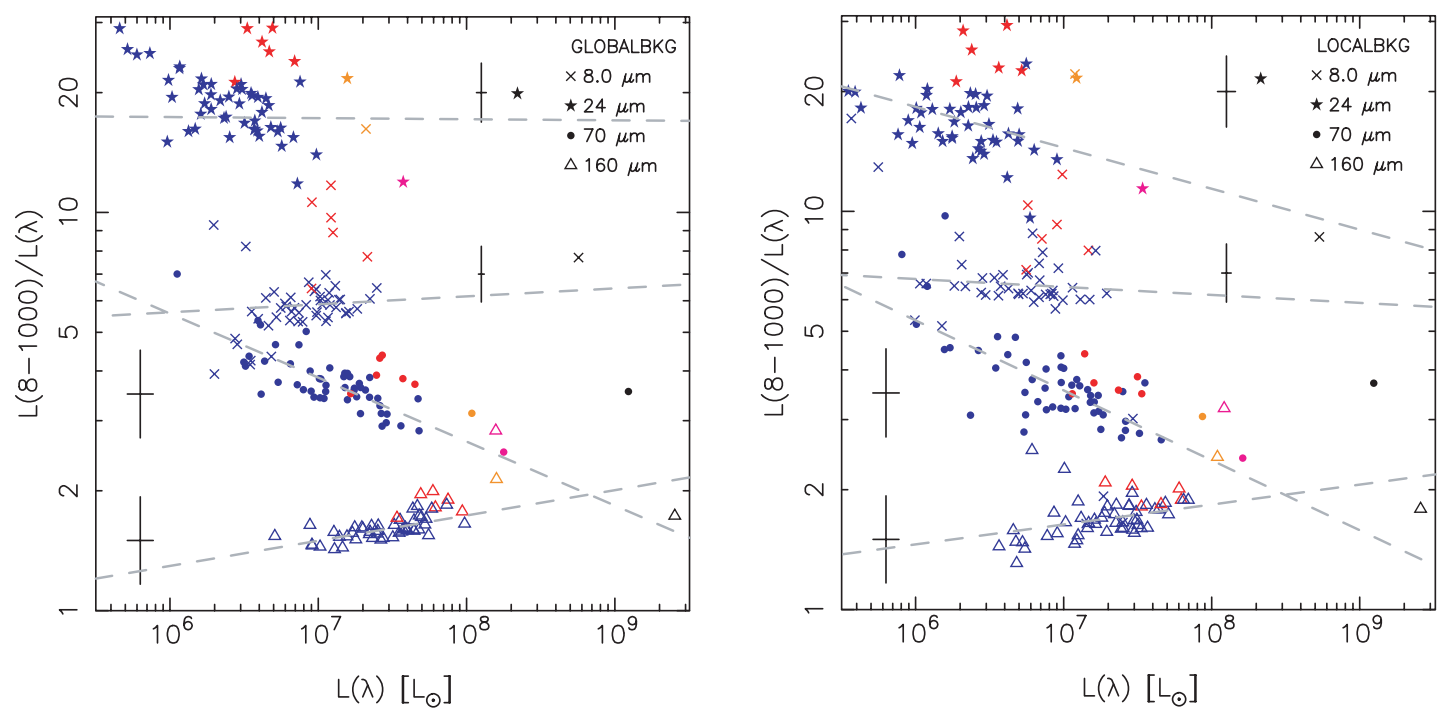

FIG. 3.- Relationship between the MIR and FIR monochromatic emissions and the $8-1000 \mu$ m luminosities for star-forming complexes in M81 (selected in 160RES resolution images). In the left panel, the data refer to the photometry calculated with a global background (GLOBALBKG case in the text). On the right, a local background (LOCALBKG case) was used to remove the diffuse component of the emission in the IR. For both panels, the integrated luminosities have been calculated by fitting models of dust emission to the SEDs for wavelengths redder than $5 \mu \mathrm{m}$. The best linear fits to the data at each wavelength are also shown. The different colors refer to the distinct types of regions defined in the left panel of Fig. 1. In both panels, average errors for the different luminosities (horizontal bars) and luminosity ratios (vertical bars) are plotted to one side of the corresponding data points. 

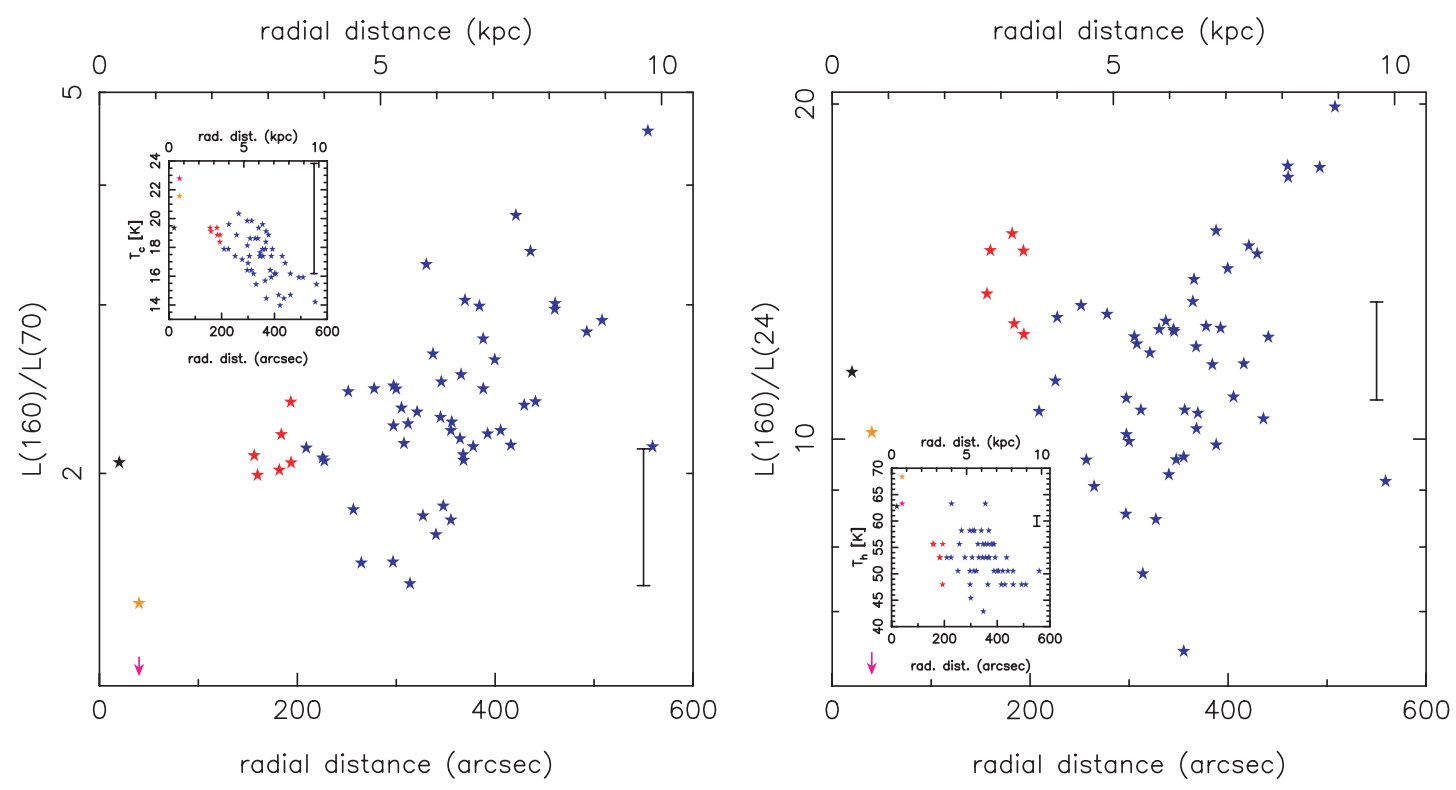

FIG. 5.- Radial plots (using deprojected radial distances) of the MIPS colors for the regions selected at 160RES. The different colors refer to the distinct types of regions defined in the left panel of Fig. 1. The average surface density of the entire galaxy has been plotted at $r=20^{\prime \prime}$, and the surface density for the nuclear and the circumnuclear regions has been plotted at $r=40^{\prime \prime}$ (slightly shifted from $r=0$ for clarity). The insets in each panel show the dust temperatures for the cold (left panel) and warm (right panel) components in our models. Average errors for the colors and temperatures are shown in each panel. 